\title{
Studies on Bio-adhesion of Matrix Tablets: I. Release Profile of Theophylline Anhydrous
}

\author{
Md. Shamsuddin ${ }^{1}$, Parvin Akter ${ }^{1}$, Md. Ziaur Rahman Khan ${ }^{2}$, \\ J akir Ahmed Chowdhury ${ }^{2}$ and Md. Selim Reza ${ }^{2}$
}

\author{
${ }^{1}$ Department of Pharmacy, The University of Asia Pacific, Dhaka-1209, Bangladesh \\ ${ }^{2}$ Faculty of Pharmacy, University of Dhaka, Dhaka-1000, Bangladesh
}

\begin{abstract}
Controlled release matrix tablets of theophylline anhydrous were designed with different types of bioadhesive polymers. HPMC 15 cps and 50 cps, Na-CMC, Gelatin, Xanthun gum and PVP K-30 were selected to formulate matrix tablets. Tablets of theophylline were prepared by direct compression method and were subjected to in vitro drug dissolution for $8 \mathrm{hrs}$ in a gastric fluid media by using thermal shaker with a shaking speed of $50 \mathrm{rpm}$ at a temperature of $37 \pm 0.5^{\circ} \mathrm{C}$. The in vitro release study as well as retention time of bioadhesive tablets on mucous membrane were investigated to develop a bioadhesive polymer based controlled release delivery system and to evaluate the performance of such delivery device. Na-CMC, HPMC and Xanthan gum based tablets showed greater bio-adhesive strength where as gelatin and PVP K-30 based tablets showed poor bioadhesive strength. Na-CMC and Xanthun gum loaded tablets were not discharged from the mucous membrane and these tablets were fully dissolved in the gastric fluid. Xanthan gum, Na-CMC and HPMC based formulation showed nearly zero-order release. On the contrary, gelatin and PVP K-30 based formulation showed a burst release within one hour of dissolution.
\end{abstract}

Key words: Bio-adhesion, Release profile, theophylline anhydrous.

\section{INTRODUCTION}

The development of bioadhesive controlled release systems has been the subject of many studies in recent years. The concept of controlled drug delivery has been traditionally used to obtain specific release rates of active ingredients. The phenomenon of bio-adhesion, introduced by Park and Robinson, has been studied extensively in the last decade and applied to improve the performance of these drug delivery systems. ${ }^{1}$ Bio-adhesion has been a topic of interest in the design of drug delivery systems to prolong the residence time of the dosage form at the site of absorption and to facilitate intimate contact of the dosage form with the underlying absorption

Correspondence to: Md. Selim Reza

Tel:880-2-8612069; Fax: 880-2-8615583

Email: selimreza_04@yahoo.com

Dhaka Univ. J. Pharm. Sci. 5(1-2): 33-37, 2006 (June-December) surface to improve and enhance the bioavailability of drugs. $^{2-5}$ In addition, bio-adhesive dosage form have been used to target local disorders at the mucosal surface to reduce the overall dosage required and minimize side-effects that may be caused by systemic administration of drugs. Bio-adhesive formulations use polymers as the adhesive component. These polymers are often water-soluble and when used in a dry form they attract water from the mucosal surface and this water transfer leads to a strong interaction. These polymers also form viscous layers when hydrated with water that increase the retention time over mucosal surfaces and may lead to adhesive interactions. ${ }^{6}$ Several studies reported bioadhesive drug delivery systems in the form of tablets, films, patches, and gels for oral, buccal, nasal, ocular, and topical routes; however, very few reports on bioadhesive microcapsules are available..$^{7-10}$ 
Prolonged contact time of a drug with a body tissue, through the use of a bioadhesive polymer can significantly improve the performance of many drugs. In our study, theophylline is used as a model drug. The objective of this study is to develop, characterize, and evaluate mucoadhesive matrix tablets of theophylline employing various bioadhesive polymers for prolonged gastrointestinal absorption.

\section{MATERIALS AND METHODS}

Theophylline anhydrous was a kind gift from Square Pharmaceuticals Bangladesh Limited. Hydroxypropylmethylcellulose (HPMC $15 \mathrm{cps}$ and $50 \mathrm{cps}$ ), Sodium carboxy methylcellulose (Na-CMC), Xanthan gum, Gelatin and PVP K-30 were gift samples from Loba chemie pvt. Ltd., India. Magnesium stearate and Aerosil were procured from Hanau chemical Ltd. (Japan). For determining bioadhesive strength, gastric mucosa of cow was obtained from a local slaughter house in Dhaka,
Bangladesh. All other reagents employed were of analytical or pharmaceutical grade.

Preparation of Matrix tablets. Theophylline matrix tablets were prepared by direct compression method using different bioadhesive polymers. The formulations of matrix tablets with their codes are presented in Table 1. In all the cases, the amount of active ingredient is $100 \mathrm{mg}$. The total weight of tablets were $406 \mathrm{mg}$ (F1a-F1f) and $609 \mathrm{mg}$ (F2a-F2f) The corresponding amount of active ingredient and HPMC-15 cps and 50 cps, Na-CMC, PVP K-30, gelatin and xanthan gum were weighed separately and mixed thoroughly in a drum blender. Matrix tablets were prepared using Perkin-Elmer laboratory hydraulic press and compression forces were 5 ton. Before compression, the surface of the die and punch were lubricated with magnesium stearate. All the theophylline loaded matrix tablets were stored in airtight containers at room temperature for further study.

Table 1. Composition of different formulations (mg) of matrix tablets.

\begin{tabular}{llllllllllllll}
\hline & F-1a & F-1b & F-1c & F-1d & F-1e & F-1f & F-2a & F-2b & F-2c & F-2d & F-2e & F-2f \\
\hline Theophylline Anhydrous & 100 & 100 & 100 & 100 & 100 & 100 & 100 & 100 & 100 & 100 & 100 & 100 \\
HPMC-15cps & 300 & - & - & - & - & - & 500 & - & - & - & - & - \\
HPMC-50cps & - & 300 & - & - & - & - & - & 500 & - & - & - & - \\
Gelatin & - & - & 300 & - & - & - & - & - & 500 & - & - & - \\
PVP K-30 & - & - & - & 300 & - & - & - & - & - & 500 & - & - & - \\
Na-CMC & - & - & - & - & 300 & - & - & - & - & - & 500 & - \\
Xanthan gum & - & - & - & - & - & 300 & - & - & - & - & - & 500 \\
Aerosil & 4 & 4 & 4 & 4 & 4 & 4 & 5 & 5 & 5 & 5 & 5 & 5 \\
Mg-Stearate & 2 & 2 & 2 & 2 & 2 & 2 & 4 & 4 & 4 & 4 & 4 & 4 \\
\hline
\end{tabular}

Dissolution studies. In vitro drug release studies of the matrix tablets were conducted in a thermal shaker (Memmert) apparatus equilibrated at temperature $37 \pm 0.5^{\circ} \mathrm{C}$ and 50 RPM speed. The dissolution studies were carried out in triplicate for 8 hrs in $250 \mathrm{ml}$ of gastric fluid (pH 1.2). The dissolution samples were collected at every 30 mins interval and replaced with an equal volume of gastric fluid to maintain the volume constant. The sample solution was analyzed at $271 \mathrm{~nm}$ as mentioned in USP and BP by an UV spectrophotometer
(Shimadzu, Japan). The amount of drug present in the samples was calculated with the help of appropriate calibration curves constructed from reference standard of respective drug. Drug dissolved at specified periods was plotted as percent release versus time (hours) curve.

Bio-adhesion testing by in vitro Wash-Off Test. The mucoadhesive property of the bioadhesive tablet was evaluated by an in vitro adhesion testing method known as the wash-off method by Lehr et 
al. ${ }^{11}$ Freshly excised pieces of intestinal mucosa $(4 \times 3$ inch) from cow were mounted on to the stainless still slide connected with a suitable support. Three tablets were placed on to wet rinsed tissue specimen and immediately there after the support was hung on to the arm of the USP tablet disintegrating test machine. When the disintegrating machine was operated, the tissue specimen was given a slow, regular up-anddown movement in the gastric fluid $(\mathrm{pH}=1.2)$ at $37^{\circ} \mathrm{C}$ contained in a one liter vessel of the machine.

\section{RESULTS AND DISCUSSION}

Effect of HPMC-15 cps and HPMC-50 cps on the release and bio-adhesive strength of Theophylline. Figure 1(A) and Figure 1(B) shows the effect of different concentration of HPMC $15 \mathrm{cps}$ and $50 \mathrm{cps}$ on theophylline anhydrous matrix tablet. A significant difference in release pattern was observed between the formulation F1 (a), F1 (b), F2 (a) and F2 (b). F1 (a) and F2 (a) contain $300 \mathrm{mg}$ and $500 \mathrm{mg}$ of HPMC-15cps and released 8 and 2.5\%, respectively after 30 mins of dissolution period and released 55.08 and $37.25 \%$ after 8 hours of dissolution period. Whereas F1 (b) and F2 (b) contain $300 \mathrm{mg}$ and $500 \mathrm{mg}$ of HPMC-50cps and released only 1.7 and $3.39 \%$ after 30 mins of dissolution study and released 51.86 and $40.57 \%$ at the end of the dissolution period. It is clearly evident that drug release was decreased with the increase of percentage polymer loading of the drug formulation. Higher viscosity of HPMC might have formed a more viscous gel like network around the delivery device which results drug release to be slowed down due to increased thickness. These gel forming agents initially formed a hydrated gel matrix which is involved in the phenomenon of bioadhesion. Three tablets were taken to observe the bio-adhesive strength. In case of F1a, all three tablets were discharged from the mucous after 16, 20 and 27 mins and as F2a contained $500 \mathrm{mg}$ of HPMC 15 cps, tablets were discharged after 23, 36 and 38 mins. Increasing the polymer amount may provide more adhesive sites and polymer chains for interpenetration with mucin, resulting consequently in the augmentation of bioadhesive strength. ${ }^{12}$ As the HPMC 50 cps tablets are of higher viscosity grade, they were not detached from the mucous membrane due to the formation of more concentrated gel layer.

Effect of Gelatin on the release and bioadhesive strength of Theophylline from F-1c \& F2c. The release profiles of Theophylline Anhydrous from F-1c and F-2c are depicted in Figure 1(C). F-1c contains 300mg of Gelatin and F-2c contains $500 \mathrm{mg}$ of Gelatin. About 2.23 and 5.6\% of Theophylline released from $\mathrm{F}-1 \mathrm{C}$ and $\mathrm{F}-2 \mathrm{c}$ respectively after 30 minutes of dissolution period. Within two hours of dissolution period F-2C showed burst release whereas F-1c released only $21.54 \%$ of theophylline. In case of $\mathrm{F}-1 \mathrm{c}$, three tablets were detached from the mucous after 10, 15 and $17 \mathrm{sec}$. respectively and tablets were also detached from the mucous within $40 \mathrm{sec}$. from F-2c. Therefore, bioadhesive strength of gelatin was very poor.

Effect of PVP K-30 on the release and bioadhesive strength of Theophylline from F-1d \& F2d. The release profile of Theophylline anhydrous from F-1d and F-2d are shown in Figure 1(D). F-1d and F-2d contains $300 \mathrm{mg}$ and $500 \mathrm{mg}$ of PVP K-30. About 5.40 and $5.48 \%$ of Theophylline released from F-1d and F-2d, respectively after 30 minutes of dissolution period. Both the formulation showed a burst release of active ingredient within first hour of dissolution period. Bioadhesive strength of PVP K-30 was not significant. Tablets were detached from the mucous membrane within a few seconds.

Effect of Na-CMC on the release and bioadhesive strength of Theophylline from F-1e \& F2e. The release profiles of Theophylline anhydrous from F-1e and F-2e are placed in Figure 1(E). F-1e and F-2e contains $300 \mathrm{mg}$ and $500 \mathrm{mg}$ of Na-CMC. About 2.21 and $4.78 \%$ of Theophylline released from F-1e and F-2e, respectively after 30 minutes of dissolution period. In general, the release pattern was found to be nearly zero-order. The contribution of Na-CMC tends to polymer swelling and erosion with an increasing trend with increase in the content of the polymer. Bio-adhesive strength of Na-CMC based tablet was significant. Two tablets of three were 
detached after 3.36 hrs and $3.50 \mathrm{hrs}$ and another tablet was not detached from the mucous membrane.
Swelling occurs in case of three tablets and after 9 hrs drugs were fully dissolved in the GI fluid.
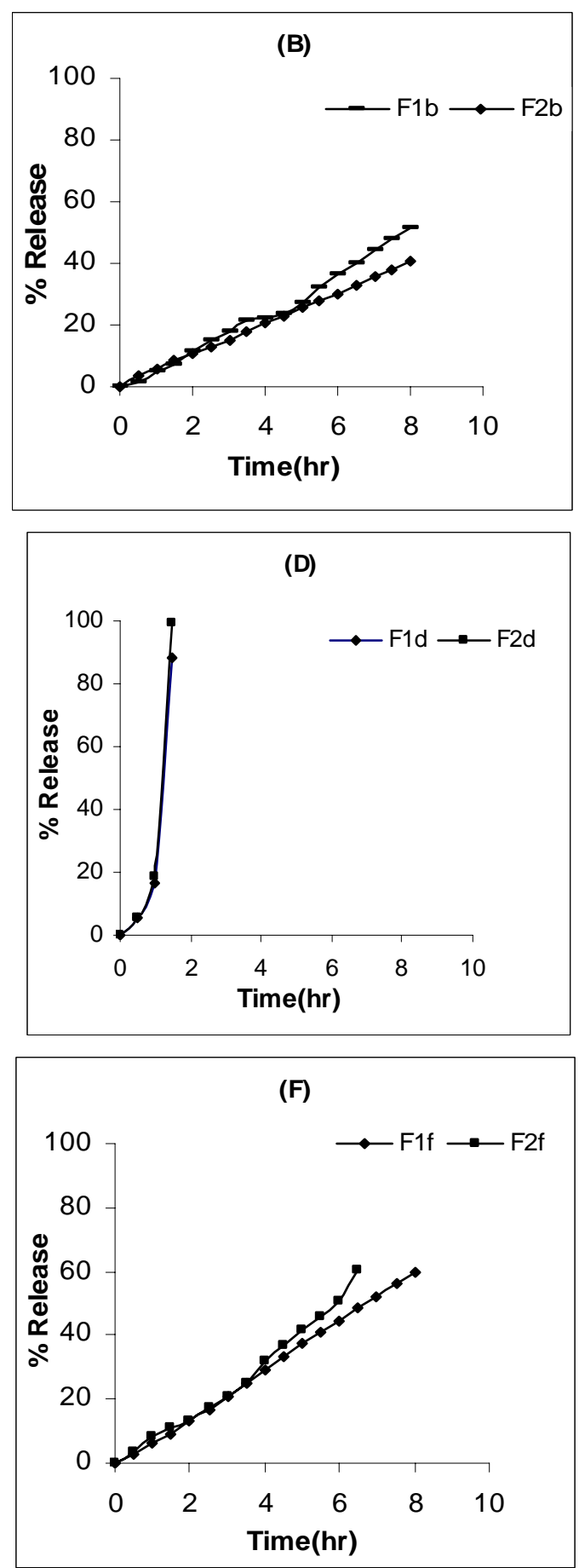

Figure 1. Release profile of theophylline anhydrous from (A) HPMC-15 cps (B) HPMC-50 cps (C) Gelatin (D) PVP K-30 (E) Na-CMC (F) Xanthan gum. 
Effect of Xanthan gum on the release and bioadhesive strength of Theophylline from F-1f and F-2f. The release profiles of Theophylline anhydrous from F-1f and F-2f are placed in Figure 1(F). F-1f and F-2f contains $300 \mathrm{mg}$ and $500 \mathrm{mg}$ of Xanthan gum. About 2.84 and $3.30 \%$ of Theophylline released from F-1f and F-2f respectively after 30 minutes of dissolution period. At the end of 8 hours of dissolution, it was found that 59.81 and $60.42 \%$ of drug released from F-1f and F$2 \mathrm{f}$ respectively. Three tablets were exposed in gastric fluid to observe the bio-adhesive strength. One tablet was discharged after 4 hrs where as other tablets were not detached from the mucous. All tablets were dissolved in gastric fluid after $10 \mathrm{hrs}$.

\section{CONCLUSION}

Bioadhesive studies and subsequent release profile were performed on matrix tablets containing theophylline anhydrous as model drug. Polymers of different hydrophylicity and bioadhesive strength were used as the matrix former. The studies showed strong bioadhesion when Na-CMC, xanthan gum and HPMC were used. However in case of gelatin and PVP K-30 no bioadhesion was observed. This is due to their inability to form viscous gel network responsible for bioadhesion capability. The release studies showed nearly zero order release pattern in case of hydrophilic polymers. In case of gelatin and PVP K-30, a burst release was observed with total breakdown of matrix within one hour of dissolution studies. From the experiment it is clearly evident that judicial use of hydrophilic polymers can increase the frequency of bioadhesion in the gastrointestinal mucous layer which will give better and prolonged chance of drug release.

\section{REFERENCES}

1. Park, K. and Robinson, J.R. 1984. Bioadhesive polymers as platforms for oral controlled drug delivery: method to study bioadhesion. Int. J. Pharm. 198, 107-127

2. Ikeda, K., Murata, K., Kobayashi, M. and Noda, K. 1992. Enhancement of bioavailability of dopamine via nasal route in beagle dogs. Chem. Pharm. Bull. 40, 2155-2158.

3. Nagai, T., Nishimoto, Y., Nambu, N., Suzuki, Y. and Sekine, K. 1984. Powder dosage forms of insulin for nasal administration. J. Control Release 1, 15-22.

4. Illum, L., Farraj, N.F., Critcheley, H. and Davis, S.S. 1988. Nasal administration of gentamicin using a novel microsphere delivery system. Int. J. Pharm. 46, 261-265.

5. Gavini, E., Sanna, V., Juliano, C., Bonferoni, M.S. and Giunchedi, P. 2002. Mucoadhesive vaginal tablets as veterinary delivery system for the controlled release of an antimicrobial drug, acriflavine. AAPS Pharm.Sci. Tech. 3: article 20 .

6. Batchelor Hannah. 2004. Novel bioadhesive formulations in drug delivery. Presented in 2004 at the British pharmaceutical conference on drug delivery to the upper GI tract, particularly the oesophagus: Medicines Research Unit, Aston University, Birmingham, B47ET, UK.

7. Sakagami, M., Kinoshita, W., Sakon, K., Sato, J.I. and Makino, Y. 2002. Mucoadhesive beclomethasone microspheres for powder inhalation: their pharmacokinetics and pharmacodynamics evaluation. J. Control Release 80, 207-218.

8. Takishima, J., Onishi, H. and Machida, Y. 2002. Prolonged intestinal absorption of cephradine with chitosan-coated ethylcellulose microparticles in rats. Biol. Pharm. Bull. 25,1498-1502.

9. Lim, S.T., Forbes, B., Berry, D.J., Martin, G.P. and Brown, M.B. 2002. In vivo evaluation of novel hyaluronan/chitosan microparticulate delivery systems for the nasal delivery of gentamicin in rabbits. Int. J. Pharm. 231, 73-82.

10. Cuna, M., Alonso, M.J. and Torres, D. 2001. Preparation and in vivo evaluation of mucoadhesive microparticles containing amoxicillin-resin complexes for drug delivery to the gastric mucosa. Eur. J. Pharm. Biopharm. 51, 199-205.

11. Lehr, C.M., Bowstra, J.A., Tukker, J.J. and Junginer, H.E. 1990. Intestinal transit of bioadhesive microspheres in an in situ loop in the rat. J. Control Release 13, 51-62.

12. Ponchel, G., Touchard, F., Duchene, D. and Peppas, N.A. 1987. Bioadhesive analysis of controlled release systems. I. Fracture and interpenetration analysis in poly (acrylic acid) containingsystems. J. Control Release 5, 129-141. 